\title{
Effects of Electric Field on the SHS Flame Propagation of the Si-C System, Examined by the Use of the Heterogeneous Theory
}

\author{
Atsushi Makino \\ Institute of Aeronautical Technology, Japan Aerospace Exploration Agency, 7-44-1 Jindaiji-Higashi, Chofu, Tokyo, Japan \\ Correspondence should be addressed to Atsushi Makino; amakino@chofu.jaxa.jp
}

Received 16 April 2013; Revised 6 August 2013; Accepted 15 August 2013

Academic Editor: Peter F. Nelson

Copyright (c) 2013 Atsushi Makino. This is an open access article distributed under the Creative Commons Attribution License, which permits unrestricted use, distribution, and reproduction in any medium, provided the original work is properly cited.

Relevant to the self-propagating high-temperature synthesis (SHS) process, an analytical study has been conducted to investigate the effects of electric field on the combustion behavior because the electric field is indispensable for systems with weak exothermic reactions to sustain flame propagation. In the present study, use has been made of the heterogeneous theory which can satisfactorily account for the premixed mode of the bulk flame propagation supported by the nonpremixed mode of particle consumption. It has been confirmed that, even for the SHS flame propagation under electric field, being well recognized to be facilitated, there exists a limit of flammability, due to heat loss, as is the case for the usual SHS flame propagation. Since the heat loss is closely related to the representative sizes of particles and compacted specimen, this identification provides useful insight into manipulating the SHS flame propagation under electric field, by presenting appropriate combinations of those sizes. A fair degree of agreement has been demonstrated through conducting an experimental comparison, as far as the trend and the approximate magnitude are concerned, suggesting that an essential feature has been captured by the present study.

\section{Introduction}

Self-propagating high-temperature synthesis (SHS) process, by virtue of a strong exothermic reaction that passes through a compacted mixture of particles, as a flame, has attracted special interests as a rapid and economical way in synthesizing inorganic and/or intermetallic compounds [1-5]. More than hundreds of kinds of materials, including borides, carbides, and silicides, are reported to be synthesized [1-5] by applying this process, while some of them are quite difficult to synthesize in conventional ways. Materials synthesized are now being considered for use as electronic materials, materials resistant to heat, corrosion, and/or wear, and so forth [1-5]. Production of functionally graded materials (FGMs) [6], composed of different components with continuous profiles, is also intended. Note also that near-net-shape fabrication can be anticipated because this is a kind of powder metallurgy.
The SHS process, applicable to various combinations of solid-solid, solid-liquid, and/or solid-gas systems, as pioneered by the group of Merzhanov and Borovinskaya [7], has also been recognized to present diverse phenomena of the flame propagation, such as pulsating, spinning, and/or repeated combustions, as well as steady propagation, as reviewed $[1-5,8,9]$. Among various systems, synthesis of Ti-C system has extensively been examined, because of its simplicity in chemical reaction, as well as its nature of refractory and hardness. It has been confirmed that not the gaseous component but the molten $\mathrm{Ti}$, spreading over carbon particles, plays an important role in the process. Effects of dominant parameters, such as the preheating, the mixture ratio of reactants, the degree of dilution, and the particle size, on the flame propagation speed and/or the range of flammability have been examined. In particular, preheating for rapid synthesis has been examined from the viewpoint of process innovation, to have higher production rates and/or 
to facilitate melting during the SHS process. Although rapid synthesis can easily be accomplished, with increasing initial temperature, a supplementary heating can sometimes cause an ignition when the compacted mixture is heated over its self- (or spontaneous) ignition temperature $[1,2,4,5,10]$, as are the cases for usual combustion.

Even for the spontaneous ignition, its utilization has been considered seriously [11-13], for those systems with weak exothermic reactions, because it is not common for those systems that sufficient preheating can be anticipated for sustaining SHS flame propagation. An external heating, by virtue of the Joule heating with applying electric fields [1420], has also been taken into account, in order to make the materials synthesis by combustion completed.

In this study, silicon carbide ( $\mathrm{SiC}$ ) has been chosen for the research subject since it has attracted special attention in the field of materials science as the light-weight materials in high-temperature structural applications, relevant to the advanced aviation gas turbines. Focus is put on the SHS flame propagation that proceeds in the compacted mixture of $\mathrm{Si}$ $\mathrm{C}$ system, from the viewpoint of combustion engineering, which has been rare, although examinations from the viewpoint of materials science have been conducted extensively [14-20]. Since self-propagation of the SHS flame cannot be anticipated without preheating and/or applying electric field, especially for the $\mathrm{Si}-\mathrm{C}$ system, here, it is intended to elucidate effects of electric field on the burning velocity and/or the limit of flammability, by the use of the heterogeneous theory [9] for the SHS process, which can satisfactorily account for the premixed mode of the bulk flame propagation supported by the nonpremixed mode of particle consumption. Experimental comparisons are also conducted, which are found to be satisfactory, as far as the trend and approximate magnitude are concerned.

\section{Formulation}

2.1. Model Definition. The problem of interest, as shown in Figure 1, is the one-dimensional, planner, heterogeneous flame propagation under electric field, being applied perpendicular to the direction of the propagation, in accordance with the experiment [19]. It is assumed in the heterogeneous theory [9] that the flame front propagates in the doubly infinite domain of a condensed medium, originally consisting of a mixture of particles of nonmetal (or higher melting-point metal) $N$, lower melting-point metal $M$, and an inert $I$ that can also be the product $P$ of the reaction between $N$ and $M$ according to

$$
v_{M} M+v_{N} N \longrightarrow v_{P} P
$$

where $v_{i}$ is the stoichiometric coefficient of the reaction. For simplicity, it is assumed that the nonmetal particlesize distribution is monodisperse, with an initial radius $R_{0}$ and number density $n_{0}$. It is also assumed that there is no reaction between $N$ and $M$ until the mixture has been heated to the melting point $T_{m}$ of the metal $M$, at which all the metal particles melt instantaneously. The reaction between the solid $N$ and molten $M$ is then assumed to take place at

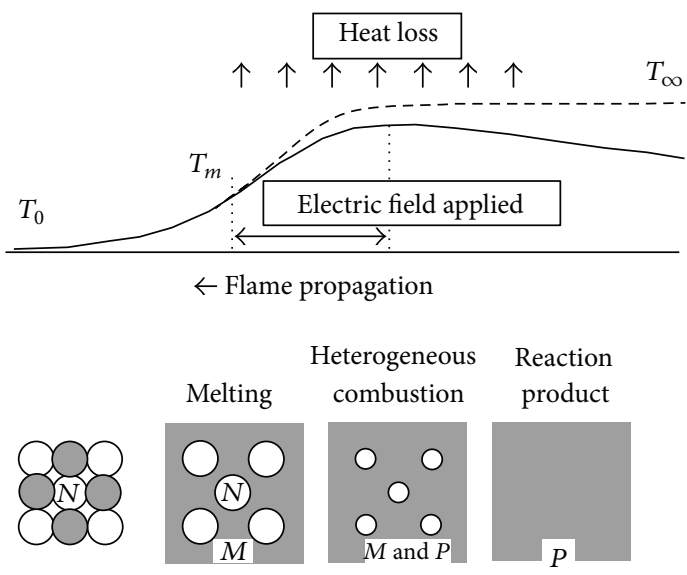

FIGURE 1: Schematic diagram of the flame structure in the propagating heterogeneous flame.

the particle surface at a finite rate and proceed until all of the $N$ particles or $M$ species are consumed. The enthalpy of phase change is neglected because it is much smaller than the heat of combustion. The lateral heat loss to the ambience, occurring throughout the entire flame structure, is also taken into account for the flame propagation considered.

2.2. Governing Equations and Boundary Conditions. By the use of the heterogeneous theory [9] for the SHS flame propagation, which can fairly formulate the situation involving a premixed mode of propagation for the bulk flame, supported by the nonpremixed reaction of the dispersed nonmetal particles in the melt, the governing equations are expressed as follows.

Continuity:

$$
\left(\rho_{t} u\right)=\left(\rho_{t} u\right)_{0}=m
$$

energy conservation:

$$
\begin{aligned}
& \frac{d}{d x}\left\{\frac{\lambda}{m} \frac{d T}{d x}-c\left(T-T_{0}\right)\right. \\
& \left.+q^{0}\left(Z-Z_{0}\right)\right\}=\frac{L-P}{m},
\end{aligned}
$$

$M$-conservation:

$$
\begin{aligned}
\left(\frac{\rho_{f} D}{m}\right) \frac{d Y_{M}}{d x}= & \left(Y_{M}+f\right) Z \\
& -\left(Y_{M, 0}+f\right) Z_{0},
\end{aligned}
$$

$N$-consumption:

$$
\frac{d(1-Z)}{d x}=-\frac{4 \pi \rho_{N} n_{0} R_{0}}{m} \frac{u_{0}}{u} \chi\left(\frac{1-Z}{1-Z_{0}}\right)^{1 / 3},
$$

where $u$ is the velocity along the $x$ coordinate, $m$ the mass burning rate (per unit area) of the flame, $T$ the temperature, $c$ the specific heat, $q^{0}$ the heat of reaction per unit mass 
of $N, Z$ the mass fraction of fluid $\left(=\rho_{f} / \rho_{t}\right), \rho_{f}$ the fluid density, $\rho_{s}$ the mass of solid per unit spatial volume, $\rho_{t}$ the total density $\left(=\rho_{f}+\rho_{s}\right), \lambda$ the thermal conductivity, $Y_{M}$ the mass fraction of $M$ in the fluid, $f$ the stoichiometric mass ratio $\left[=\left(v_{M} W_{M}\right) /\left(v_{N} W_{N}\right)\right], W$ the molecular weight, and the subscript 0 designates the unburned state.

The surface regression rate $\chi$, defined by $d R / d t=-\chi / R$, is given [9] as

$$
\chi=\left(\frac{\rho_{M} D}{\rho_{N}}\right) \tilde{\chi} ; \quad \tilde{\chi}=\ln \left[1+\left(\frac{A}{1+A}\right) \frac{Y_{M}}{f}\right],
$$

where $A=D a \exp (-T a / T), D a=(B R) / D, B$, and $T a$ are, respectively, the reduced Damköhler number, the Damköhler number, the frequency factor, and the activation temperature of the surface reaction. Note here that the nondimensional surface regression rate in (6) is presented by the use of the transfer number in terms of the natural logarithmic term, just like that for the droplet combustion, and that the transfer number here is expressed as that similar to the well-known expression for the combustion rate of solid for the first-order kinetics, suggesting that roles of diffusion and chemical kinetics are both included in the present formulation. In addition, $D$ is the Arrhenius mass diffusivity $\left[=D_{0} \exp \left(-T_{d} / T\right)\right]$ with an activation temperature $T_{d}$, being anticipated to increase markedly over a relatively thin, high temperature combustion zone, under the condition that the diffusion-limited situation prevails in the chemical kinetics at high temperatures.

A set of these governing equations is basically the same as that in the previous works [9], except for the term that appears in the energy conservation equation, in order to take account of an effect of electric field. While the heat-loss rate $L$, as expressed in the previous works, is given as

$$
L=4 \varepsilon \sigma_{\mathrm{SB}}\left(T^{2}+T_{0}^{2}\right)\left(T+T_{0}\right)\left(T-T_{0}\right) \frac{1}{2 r},
$$

where $\varepsilon$ is the emissivity and $\sigma_{\mathrm{SB}}$ is the Stefan-Boltzmann constant, the energy supply rate $P$ due to the electric field is expressed as

$$
P=(1-Z) \sigma_{c} E^{2},
$$

where $E$ is the electric field $(\mathrm{V} / \mathrm{m})$ and $\sigma_{c}$ is the electric conductivity $\left(\Omega^{-1} \mathrm{~m}^{-1}\right)$, with the Joule heating being taken into account. In accordance with experimental observations $[17,19,20]$ that the Joule heating due to external energy supply has mainly been conducted in the combustion zone, here, it is a priori assumed that it can be made by virtue of the conductible, nonmetal $N$ particles in the melt within the combustion zone, so that the mass ratio $(1-Z)$ appears in (8). It is also assumed that no energy supplies before arrival of the SHS flame can be expected, because of the extremely high resistance of contact among particles, prior to the appearance of melt. After completion of the materials synthesis, we do not expect any energy supplies, because of the high electric resistance of $\mathrm{SiC}$, which is too high to allow for the electric current.
By introducing nondimensional variables and parameters as

$$
\begin{gathered}
\sigma=\frac{\left(Z_{\infty}-Z_{0}\right) m_{a}}{(\lambda / c)} x, \quad \theta=\frac{T-T_{0}}{T_{\infty}-T_{0}}, \\
\xi=\frac{Z-Z_{0}}{Z_{\infty}-Z_{0}}, \quad L e_{0}=\frac{(\lambda / c)}{\left(\rho_{t} D\right)_{0}}, \\
\Lambda_{0}=\left[\frac{\left(Z_{\infty}-Z_{0}\right)^{2} m_{a}^{2}}{4 \pi\left(\rho_{M} D_{0}\right)(\lambda / c) n_{0} R_{0}}\right], \\
\Psi=\left(\frac{\lambda}{\rho_{t} c}\right)\left[\frac{4 \varepsilon \sigma_{\mathrm{SB}}\left(T^{2}+T_{0}^{2}\right)\left(T+T_{0}\right)}{\left(\rho_{t} c\right)\left(u_{0, a} R_{0}\right)^{2}}\right]\left(\frac{R_{0}^{2}}{2 r}\right), \\
H=\frac{(\lambda / c) \sigma_{c} E^{2}}{m_{a}^{2} q^{0}},
\end{gathered}
$$

the governing equations are as follows:

$$
\begin{aligned}
& \frac{d}{d \sigma}\left(\frac{d \theta}{d \sigma}-\frac{m / m_{a}}{Z_{\infty}-Z_{0}} \theta+\frac{m / m_{a}}{\widetilde{T}_{\infty}-\widetilde{T}_{0}} \xi\right) \\
& =\frac{\Psi \cdot \theta}{\left(Z_{\infty}-Z_{0}\right)^{2}}-\frac{H \cdot(1-\xi)}{\left(Z_{\infty}-Z_{0}\right)\left(\widetilde{T}_{\infty}-\widetilde{T}_{0}\right)}, \\
& \frac{d \xi}{d \sigma}=\frac{\tilde{\chi}}{\left(m / m_{a}\right) \Lambda_{0}\left(\rho_{t, 0} / \rho_{t}\right) \exp \left(\widetilde{T}_{d} / \widetilde{T}\right)} \\
& \quad \times\left\{1-\left(\frac{Z_{\infty}-Z_{0}}{1-Z_{0}}\right) \xi\right\}^{1 / 3} .
\end{aligned}
$$

In the above, $m_{a}$ and $\Lambda_{0}$ are, respectively, the mass burning rate and the mass burning rate eigenvalue in the adiabatic condition. The nondimensional temperature is defined as $\widetilde{T}=c_{p} T / q^{0}$. Note here that $\Psi$, called the heat-loss parameter and defined as a ratio of the heat-loss and heat-release rates, depends not only on the physicochemical parameters but also on the particle diameter $2 R_{0}$ and representative size $2 r$ of a sample specimen [9]. As for the parameter $H$ introduced here, we shall call it a heat-input parameter, hereafter.

Equations (9) through (11), together with the mass burning rate $m$, are to be solved, subject to the boundary conditions

$$
\begin{aligned}
& \sigma=0: \theta=\theta_{m}, \quad \xi=0, \\
& \sigma \longrightarrow \infty: \frac{d \theta}{d \sigma}=-\frac{\Psi \cdot \theta}{\left(m / m_{a}\right)\left(Z_{\infty}-Z_{0}\right)}, \quad \frac{d \xi}{d \sigma}=0 .
\end{aligned}
$$

Note that the cold boundary difficulty is eliminated by specifying the reaction to be initiated at the melting point of $M$. Thus, the present problem is reduced to be an eigenvalue problem for obtaining $m$ as an eigenvalue, under a nonadiabatic condition in which there exists not only heat loss but also external energy supply. 
2.3. Burning Velocity under Adiabatic Condition. When there is neither heat loss nor external energy supply, the burning velocity is obtained as [9]

$$
u_{0} R_{0}=\frac{1-Z_{0}}{Z_{\infty}-Z_{0}} D_{0} \sqrt{3 \Lambda_{0} L e_{0} \frac{\rho_{M} / \rho_{N}}{1-Z_{0}}}
$$

once the specific value of the eigenvalue $\Lambda_{0}$ is determined by conducting a numerical calculation. It is seen that the burning velocity $u_{0}$ is inversely proportional to the particle diameter $2 R_{0}$. It may be informative to note that the product of $u_{0}$ and $R_{0}$ has even been called the SHS rate-constant [9]. The mass ratio of the lower melting-point metal $M$, before and after the combustion, is expressed as

$$
\begin{gathered}
1-Z_{0}=\frac{\mu(1-\kappa)}{\mu+f}, \\
Z_{\infty}= \begin{cases}1 & (\mu \leq 1) \\
Z_{0}+\frac{1-Z_{0}}{\mu} & (\mu \geq 1) .\end{cases}
\end{gathered}
$$

Here, $\mu$ is the mixture ratio, defined as the initial molar ratio of $N$ to $M$ divided by the corresponding stoichiometric molar ratio; $\kappa$ is the degree of dilution, defined as the initial mass fraction of diluent.

2.4. Parameters. Values of physicochemical parameters employed are those of the Si-C system: $q^{0}=6.23 \mathrm{MJ} / \mathrm{kg}, c=$ $1 \mathrm{~kJ} /(\mathrm{kg} \cdot \mathrm{K}), \rho_{M}=2.34 \times 10^{3} \mathrm{~kg} / \mathrm{m}^{3}, \rho_{N}=2.25 \times 10^{3} \mathrm{~kg} / \mathrm{m}^{3}$, $W_{M}=28.1 \times 10^{-3} \mathrm{~kg} / \mathrm{mol}, W_{N}=12.0 \times 10^{-3} \mathrm{~kg} / \mathrm{mol}$, and $T_{m}=1681 \mathrm{~K}$. Although some of the chemical kinetic data are reported in [17], it is not the chemical kinetics but the mass diffusivity, at high temperatures, as explained in Section 2.2. Therefore, use has been made of the mass diffusivity, $D=3.3 \times 10^{-5} \exp \left(-3.39 \times 10^{4} / T\right) \mathrm{m}^{2} / \mathrm{s}[21]$, so that the representative Lewis number is set to be $L e_{0}=$ 0.36 . The total density before and after the combustion is assumed to be equal. Note that the thermophysical properties used here implicitly account for effects of compact density, gases in void spaces, gas evolution, and so forth. Other thermophysical properties, indispensable for examining the limit of flammability, related to the heat loss, are $\lambda /(\rho c)=$ $1.2 \times 10^{-6} \mathrm{~m}^{2} / \mathrm{s}$ for the thermometric conductivity [22] and $\varepsilon=0.8$ for the emissivity [23]. In addition, use has been made of the electric conductivity of carbon given by

$$
\sigma_{c}=1.21 \times 10^{4}+6.25\left(T_{r}-450\right) \quad\left[\Omega^{-1} \mathrm{~m}^{-1}\right],
$$

being obtained by the use of a curve fitting of data in the literature [24], as a function of the representative temperature $T_{r}$, defined as an arithmetic mean of the melting point of $\mathrm{Si}$ and the maximum flame temperature.

\section{Results}

3.1. Effects of Heat-Input Parameter in the Adiabatic Condition. First, effects of heat-input parameter on the mass burning rate are examined in the adiabatic condition. Here, mass burning rate under electric field, $m_{a, H}$, has been normalized by the use of that without electric field, $m_{a, 0}$. Figure 2 shows the normalized mass burning rate $m_{a, H} / m_{a, 0}$ for the stoichiometric Si-C system, as a function of the heat-input parameter $H$, with the initial temperature $T_{0}$ taken as a parameter. With increasing heat-input parameter $H$, the normalized mass burning rate increases first gradually and then rapidly, up to the limiting value determined in (28), to be mentioned later. It is seen that external energy supply is effective in enhancing mass burning rate and that its contribution to $m_{a, H} / m_{a, 0}$ becomes large with increasing initial temperature $T_{0}$.

3.2. Effects of Heat-Input Parameter under Heat Loss Condition. Effects of heat loss on the mass burning rate are then examined. In this examination, mass burning rate is normalized by the adiabatic mass burning rate. Figure 3 shows the normalized mass burning rate $m / m_{a}$, as a function of the heat-loss parameter $\Psi$, with the heat-input parameter $H$ taken as a parameter. When there is no electric field $(H=0)$, the well-known, characteristic extinction turningpoint behavior is exhibited, with the upper branch being the stable solution and the turning point designating the state of extinction [9]. In contrast, the trend becomes quite different from this, with increasing heat-input parameter $H$. When $H=0.1$, although there exists the turningpoint behavior, the solution ceases to exist at certain $\Psi$ and $\left(\mathrm{m} / \mathrm{m}_{a}\right)_{H}$, because of other restrictions. When $H=0.2$ or more, there appears the limit of flammability, designated by an open circle, before reaching the turning point. It is seen that, with increasing $H$, the range of $m / m_{a}$ for the flame propagation contracts, while the range of $\Psi$ expands.

Relevant to the limit of flammability under electric field, an attempt has been made to examine a range for the existence of SHS flame, which yields a relation in (29), to be mentioned later. In Figure 3, those limits are also shown by dotted curves, below which the steady propagation ceases to exist. It should be noted that, in determining the position of the SHS flame, theoretical consideration in the next section is indispensable.

\section{Theoretical Consideration}

4.1. Flame Position and Temperature. In the limit of large Zeldovich number, melting, diffusion, and consumption/ convection are confined to a thin layer in the neighborhood of the SHS flame. Outside this layer, the diffusion and consumption/convection terms are exponentially small. If we consider the situation that the flame locates at $\sigma=\sigma_{F}$ and that the electric field is supplied in the region $0<\sigma<\sigma_{F}$, temperature profile outside the flame is expressed as

$$
\begin{aligned}
& \sigma \leq 0: \theta=A_{u} \exp \left(\lambda_{u} \sigma\right), \quad \xi=0, \\
& 0<\sigma<\sigma_{F}: \theta=A_{h} \exp \left(\lambda_{u} \sigma\right)+B_{h} \exp \left(\lambda_{d} \sigma\right) \\
& +\frac{H \cdot\left(Z_{\infty}-Z_{0}\right)}{\Psi \cdot\left(\widetilde{T}_{\infty}-\widetilde{T}_{0}\right)}, \quad \xi=0, \\
& \sigma>\sigma_{F}: \theta=C \exp \left(\lambda_{d} \sigma\right), \quad \xi=1,
\end{aligned}
$$




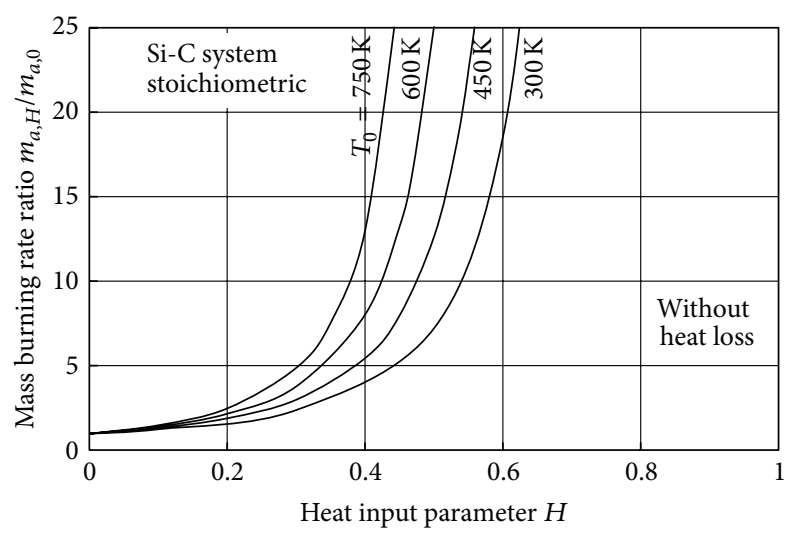

FIGURE 2: Normalized mass burning rate $m_{a, H} / m_{a, 0}$ for the stoichiometric $\mathrm{Si}-\mathrm{C}$ system as a function of the heat-input parameter $H$, with the initial temperature $T_{0}$ taken as a parameter when there exists no heat loss in the SHS flame propagation.

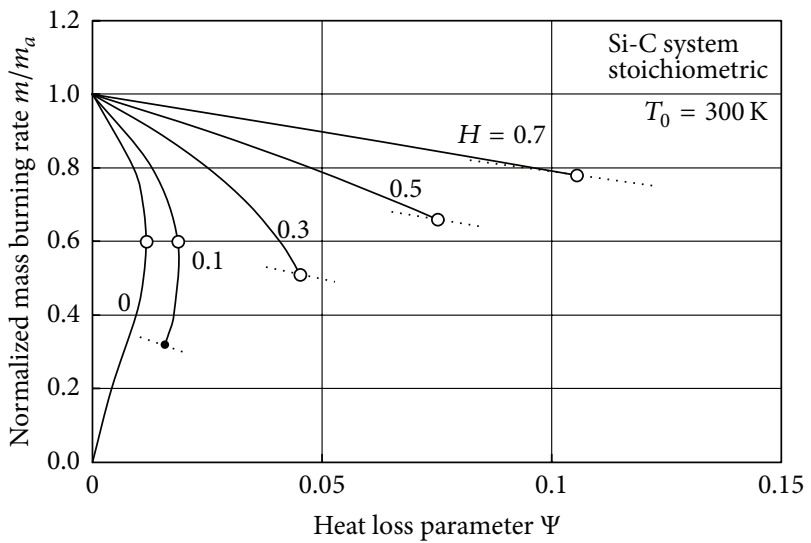

FIGURE 3: Normalized mass burning rate $m / m_{a}$ for the stoichiometric Si-C system as a function of the heat loss parameter $\Psi$, with the heat-input parameter $H$ taken as a parameter. An open circle designates the limit of flammability.

where

$$
\begin{aligned}
& \lambda_{u}=\frac{m / m_{a}}{2\left(Z_{\infty}-Z_{0}\right)}\left\{1+\sqrt{1+\frac{4 \Psi}{\left(m / m_{a}\right)^{2}}}\right\}, \\
& \lambda_{d}=\frac{m / m_{a}}{2\left(Z_{\infty}-Z_{0}\right)}\left\{1-\sqrt{1+\frac{4 \Psi}{\left(m / m_{a}\right)^{2}}}\right\} .
\end{aligned}
$$

Relations among constants $A_{u}, A_{h}, B_{h}$, and $C$ are determined by the use of the continuity in temperature and heat flux at the position $\sigma=0$, the continuity in temperature at the flame position $\left(\sigma=\sigma_{F}\right)$, and the jump condition at the flame:

$$
\left(\frac{d \theta}{d \sigma}\right)_{\sigma_{F-}}-\left(\frac{d \theta}{d \sigma}\right)_{\sigma_{F+}}=\frac{m / m_{a}}{\widetilde{T}_{\infty}-\widetilde{T}_{0}}
$$

Then, we have

$$
\begin{aligned}
A_{u}= & \frac{1}{\lambda_{u}-\lambda_{d}} \frac{m / m_{a}}{\widetilde{T}_{\infty}-\widetilde{T}_{0}} \exp \left(-\lambda_{u} \sigma_{F}\right) \\
& -\frac{\lambda_{d}}{\lambda_{u}-\lambda_{d}} \frac{H \cdot\left(Z_{\infty}-Z_{0}\right)}{\Psi \cdot\left(\widetilde{T}_{\infty}-\widetilde{T}_{0}\right)}\left[1-\exp \left(-\lambda_{u} \sigma_{F}\right)\right], \\
A_{h}= & \left\{\frac{1}{\lambda_{u}-\lambda_{d}} \frac{m / m_{a}}{\widetilde{T}_{\infty}-\widetilde{T}_{0}}\right. \\
& \left.+\frac{\lambda_{d}}{\lambda_{u}-\lambda_{d}} \frac{H \cdot\left(Z_{\infty}-Z_{0}\right)}{\Psi \cdot\left(\widetilde{T}_{\infty}-\widetilde{T}_{0}\right)}\right\} \exp \left(-\lambda_{u} \sigma_{F}\right), \\
C= & \frac{1}{\lambda_{u}-\lambda_{d}} \frac{m / m_{\infty}}{\widetilde{T}_{\infty}-\widetilde{T}_{0}} \exp \left(-\lambda_{d} \sigma_{F}\right) \\
& -\frac{\lambda_{u}=\left(Z_{\infty}-Z_{0}\right)}{\lambda_{u}-\lambda_{d}} \frac{H \cdot\left(Z_{\infty}-Z_{0}\right)}{\Psi \cdot\left(\widetilde{T}_{\infty}-\widetilde{T}_{0}\right)}\left[1-\exp \left(-\lambda_{d} \sigma_{F}\right)\right] .
\end{aligned}
$$

If we further note that the temperature at $\sigma=0$ is the melting point $T_{m}$, we can determine the flame position $\sigma_{F}$ as

$$
\sigma_{F}=\frac{1}{\lambda_{u}} \ln \left[\frac{\left(m / m_{a}\right)+\left(\lambda_{d} / \Psi\right) H \cdot\left(Z_{\infty}-Z_{0}\right)}{\left(\widetilde{T}_{m}-\widetilde{T}_{0}\right)\left(\lambda_{u}-\lambda_{d}\right)+\left(\lambda_{d} / \Psi\right) H \cdot\left(Z_{\infty}-Z_{0}\right)}\right],
$$

by the use of (21). The flame temperature $T_{\infty}$ in the adiabatic condition $(\Psi=0)$, which is indispensable for nondimensionalization, is obtained from (24) as

$$
\left(\widetilde{T}_{\infty}-\widetilde{T}_{0}\right)=\left(Z_{\infty}-Z_{0}\right)\left(1+\frac{H \cdot \sigma_{F}}{Z_{\infty}-Z_{0}}\right)
$$

$$
\frac{\sigma_{F}}{Z_{\infty}-Z_{0}}=\ln \left[\frac{(1-H)\left(Z_{\infty}-Z_{0}\right)}{\left(\widetilde{T}_{m}-\widetilde{T}_{0}\right)-H \cdot\left(Z_{\infty}-Z_{0}\right)}\right] \text {. }
$$

We see that the adiabatic flame temperature is increased due to the application of the electric field.

4.2. Range of Electric Field. Even though the electric field is effective in sustaining SHS flame propagation, there exists an indispensable requirement for the flame position to be positive $\left(\sigma_{F} \geq 0\right)$, which further requires that the logarithmic term in (27) should be positive. From this requirement, we can determine the range for the valid heat-input parameter $H$ as

$$
0 \leq H<\frac{\widetilde{T}_{m}-\widetilde{T}_{0}}{Z_{\infty}-Z_{0}}<1
$$


In the same manner, when there exists heat loss, we have from (25) the following relation between the heat-loss parameter $\Psi$ and the heat-input parameter $H$ :

$$
\begin{aligned}
0 \leq & \Psi<-\frac{1}{2}\left\{\frac{\left(m / m_{a}\right)^{2}}{4}-\frac{H\left(Z_{\infty}-Z_{0}\right)}{\widetilde{T}_{m}-\widetilde{T}_{0}}\right\} \\
& +\sqrt{\frac{\left(m / m_{a}\right)^{2}}{8}\left\{\frac{\left(m / m_{a}\right)^{2}}{8}+\frac{H\left(Z_{\infty}-Z_{0}\right)}{\widetilde{T}_{m}-\widetilde{T}_{0}}\right\}} .
\end{aligned}
$$

\section{Experimental Comparison}

Although an introduction of the heat input parameter $H$ has turned out to be useful in examining the limit of flammability, effects of electric field $E$ on the mass burning rate $m_{a}$ are to be examined directly because use has been made of $m_{a}$ in defining $H$, as shown in (12). Figure 4 shows the SHS rate-constant $u_{0} R_{0}$ as a function of the electric field $E$, with the initial temperature $T_{0}$ taken as a parameter. The SHS rate-constant $u_{0} R_{0}$ expressed in (15) is used, instead of the normalized mass burning rate used in Figures 2 and 3. It is seen that the SHS rate-constant $u_{0} R_{0}$ in the adiabatic condition, shown by solid curves, increases gradually with an increase in the electric field $E$, because of the external energy supply. It is also seen that, at a certain electric field, the SHS rate-constant $u_{0} R_{0}$ becomes high, with increasing initial temperature $T_{0}$, because of the preheating effect.

A dashed curve in Figure 4 shows the limit of flammability. As the representative size of the cross-sectional area in the compacted specimen, indispensable in determining heatloss parameter $\Psi$ in (11), use has been made of $9.175 \mathrm{~mm}$ for $2 r$, determined by use of the relation for a rectangular crosssection as [9]

$$
\ell=\frac{2 a b}{a+b}
$$

with $a=14.1 \mathrm{~mm}$ and $b=6.8 \mathrm{~mm}$, in accordance with the experiment by Feng and Munir [19]. As for the particle size of carbon, use has been made of $2 R_{0}=5 \mu \mathrm{m}$, reported. Although the limit of flammability, shown in Figure 4, is only that for $T_{0}=300 \mathrm{~K}$, it has been confirmed in a preparatory study that it is nearly independent of the initial temperature $T_{0}$, within the range examined.

Data points in Figure 4 are experimental in the literature [19]; a symbol ( $\square$ ) designates a result for relative density 0.64 and (o) for relative density 0.52 . It is seen that there is no remarkable effect of the relative density and that a fair degree of agreement exists between experimental and theoretical results, as far as the trend and approximate magnitude are concerned. It is also seen that the SHS flame propagation under low electric fields, say, less than about $1000 \mathrm{~V} / \mathrm{m}$, proceeds close to the limit of flammability at $T_{0}=300 \mathrm{~K}$. To the contrary, at high electric fields, the SHS flame propagation proceeds under the condition, free from heat loss, because of the external energy supply.

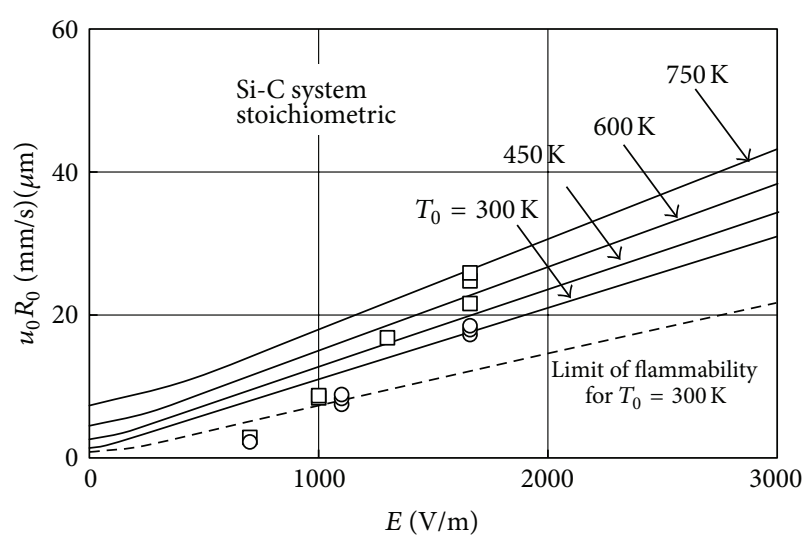

FIGURE 4: SHS rate-constant $u_{0} R_{0}$ as a function of the electric field $E$, with the initial temperature $T_{0}$ taken as a parameter. Solid curves represent the state when there is no heat loss; a dashed curve represents the limit of flammability. Data points are experimental [19].

\section{Concluding Remarks}

In the present study, flame propagation in the SHS process of the Si-C system has been examined by the use of the heterogeneous theory, with taking account of an effect of electric field. It has been shown that the burning velocity is enhanced by applying an electric field, due to an increase in the external energy supply, as reported in the literature. Furthermore, it has been confirmed that there exists the limit of flammability, even for the SHS flame propagation under electric field, as is the case for usual SHS flame propagation, although its dependence on the heat-loss rate is quite different from that without electric field. A fair degree of agreement between experimental and theoretical results suggests that the present study has captured the essential feature of the SHS flame propagation under electric field, providing useful insight into manipulating the SHS flame propagation, by the use of representative sizes of particles and compacted specimen, which has not been recognized in the previous studies.

\section{Nomenclature}

A: Constant for temperature profile

$a$ : Length of a rectangular sample specimen [m]

B: Constant for temperature profile

$b$ : Width of a rectangular sample specimen [m]

C: Constant for temperature profile

c: Specific heat $[\mathrm{J} /(\mathrm{kg} \cdot \mathrm{K})]$

$D:$ Diffusivity $\left[\mathrm{m}^{2} / \mathrm{s}\right]$

E: Electric field $[\mathrm{V} / \mathrm{m}]$

$f$ : Stoichiometric mass $\operatorname{ratio}\left[=\left(v_{M} W_{M}\right) /\left(v_{N} W_{N}\right)\right]$

$H$ : Heat input parameter

$L:$ Heat loss $\left[\mathrm{J} /\left(\mathrm{m}^{3} \cdot \mathrm{s}\right)\right]$

Le: Lewis number

$\ell$ : Representative size of the rectangular sample specimen [m] 
$m$ : Mass burning rate $\left[\mathrm{kg} /\left(\mathrm{m}^{2} \cdot \mathrm{s}\right)\right]$

$P: \quad$ Energy supply due to electric field $\left[\mathrm{J} /\left(\mathrm{m}^{3} \cdot \mathrm{s}\right)\right]$

$q^{0}$ : Heat of combustion per unit mass of $N[\mathrm{~J} / \mathrm{kg}]$

$R$ : Particle radius [m]

$r$ : Radius of sample specimen [m]

$T$ : Temperature [K]

$T_{d}$ : Activation temperature of mass diffusivity $[\mathrm{K}]$

$u$ : Burning velocity $[\mathrm{m} / \mathrm{s}]$

$W$ : Molecular weight

$Y$ : Mass fraction

$Z$ : Mass ratio of species $M$.

\section{Greek}

$\varepsilon: \quad$ Emissivity

$\theta$ : Nondimensional temperature

$\kappa$ : Degree of dilution

$\Lambda$ : Eigenvalue of the mass burning rate

$\lambda$ : Thermal conductivity $[\mathrm{W} /(\mathrm{m} \cdot \mathrm{K})]$ or exponent for temperature profile

$\mu$ : $\quad$ Mixture ratio

$v$ : Stoichiometric coefficient

$\xi$ : Normalized mass ratio of $M$

$\sigma:$ Nondimensional length

$\sigma_{c}:$ Electric conductivity $\left[\Omega^{-1} \mathrm{~m}^{-1}\right]$

$\sigma_{\mathrm{SB}}$ : Stefan-Boltzmann constant $\left[\mathrm{W} /\left(\mathrm{m}^{2} \cdot \mathrm{K}^{4}\right)\right]$

$\rho:$ Density $\left[\mathrm{kg} / \mathrm{m}^{3}\right]$

$\chi$ : Surface regression rate $\left[\mathrm{m}^{2} / \mathrm{s}\right]$

$\Psi$ : Heat loss parameter.

\section{Subscript}

a: Adiabatic condition

d: Downstream

F: Flame

$f$ : $\quad$ Fluid or melt

$H$ : Electric field

$h$ : Hot zone

$M$ : Lower melting-point metal

$m$ : Melting point

$N$ : Nonmetal or higher melting-point metal

$P$ : Combustion product or diluent

$s$ : Solid or surface

$t$ : Total

$u$ : Upstream

0: Initial or unburned state.

\section{Superscript}

$\sim$ : Nondimensional or stoichiometrically mass weighted.

\section{References}

[1] W. L. Frankhouser, K. W. Brendley, M. C. Kieszek, and S. T. Sullivan, Gasless Combustion Synthesis of Refractory Compounds, Noyes, Park Ridge, NJ, USA, 1985.
[2] Z. A. Munir and U. Anselmi-Tamburini, "Self-propagating exothermic reactions: the synthesis of high-temperature materials by combustion," Materials Science Reports, vol. 3, no. 7-8, pp. 277-365, 1989.

[3] A. G. Merzhanov, Self-Propagating High-Temperature Synthesis: Twenty Years of Search and Findings, Combustion and Plasma Synthesis of High-Temperature Materials, VCH Publishers, New York, NY, USA, 1990, Edited by Z. A. Munir and J. B. Holt.

[4] A. Varma and J.-P. Lebrat, "Combustion synthesis of advanced materials," Chemical Engineering Science, vol. 47, no. 9-11, pp. 2179-2194, 1992.

[5] A. Varma, A. S. Rogachev, A. S. Mukasyan, and S. Hwang, "Combustion synthesis of advanced materials: principles and applications," Advances in Chemical Engineering, vol. 24, no. C, pp. 79-226, 1998.

[6] W. G. J. Bunk, Gradient Materials For Structural and Functional Applications, Advanced Materials '93, III/B, Elsevier, Amsterdam, The Netherlands, 1994.

[7] A. G. Merzhanov and I. P. Borovinskaya, "A new class of combustion processes," Combustion Science and Technology, vol. 10, no. 5-6, pp. 195-201, 1975.

[8] S. B. Margolis, "The transition to nonsteady deflagration in gasless combustion," Progress in Energy and Combustion Science, vol. 17, no. 2, pp. 135-162, 1991.

[9] A. Makino, "Fundamental aspects of the heterogeneous flame in the self-propagating high-temperature synthesis (SHS) process," Progress in Energy and Combustion Science, vol. 27, no. 1, pp. 1-74, 2001.

[10] A. G. Merzhanov, "Nonisothermal methods in chemical kinetics," Combustion, Explosion, and Shock Waves, vol. 9, no. 1, pp. 3-28, 1975.

[11] A. S. Rogachev, A. S. Mukasyan, and A. Varma, "Volume combustion modes in heterogeneous reaction systems," Journal of Materials Synthesis and Processing, vol. 10, no. 1, pp. 31-36, 2002.

[12] J. P. Lebrat, A. Varma, and A. E. Miller, "Combustion synthesis of $\mathrm{Ni}_{3} \mathrm{Al}$ and $\mathrm{Ni}_{3} \mathrm{Al}$-matrix composites," Metallurgical Transactions A, vol. 23, no. 1, pp. 69-76, 1992.

[13] L. Thiers, A. S. Mukasyan, and A. Varma, "Thermal explosion in Ni-Al system: influence of reaction medium microstructure," Combustion and Flame, vol. 131, no. 1-2, pp. 198-209, 2002.

[14] V. A. Knyazik, A. G. Merzhanov, V. B. Solomonov, and A. S. Shteinberg, "Macrokinetics of high-temperature titanium interaction with carbon under electrothermal explosion conditions," Combustion, Explosion, and Shock Waves, vol. 21, no. 3, pp. 333337, 1985.

[15] O. Yamada, Y. Miyamota, and M. Koizumi, "Self-propagating high-temperature synthesis of the SiC," Journal of Materials Research, vol. 1, no. 2, pp. 275-279, 1986.

[16] V. A. Knyazik, A. G. Merzhanov, and A. S. Shteinberg, "About combustion mechanism of the Ti-C system," Doklady Akademii Nauk SSSR, vol. 301, no. 3, pp. 899-902, 1989.

[17] V. I. Gorovenko, V. A. Knyazik, and A. S. Shteinberg, "Hightemperature interaction between silicon and carbon," Ceramics International, vol. 19, no. 2, pp. 129-132, 1993.

[18] A. Feng and Z. A. Munir, "The effect of an electric field on self-sustaining combustion synthesis: part I. modeling studies," Metallurgical and Materials Transactions B, vol. 26, no. 3, pp. 581-586, 1995.

[19] A. Feng and Z. A. Munir, "The effect of an electric field on selfsustaining combustion synthesis: part II. field-assisted synthesis 
of $\beta$-SiC," Metallurgical and Materials Transactions B, vol. 26, no. 3, pp. 587-593, 1995.

[20] A. Feng and Z. A. Munir, "Relationship between field direction and wave propagation in activated combustion synthesis," Journal of the American Ceramic Society, vol. 79, no. 8, pp. 20492058, 1996.

[21] Thermophysical Properties Handbook, Japan Society of Thermophysical Properties, Yokendo, Tokyo, Japan, 1990.

[22] Thermophysical Properties Handbook, Japan Society of Thermophysical Properties, Yokendo, Tokyo, Japan, 1990.

[23] S. Hanzawa, "Refractories of furnaces to reduce environmental impact," Annual Report of Ceramics Research Laboratory, vol. 9, pp. 33-42, 2009 (Japanese).

[24] Thermophysical Properties of High Temperature Solid Materials, vol. 1, MacMillan, New York, NY, USA, 1965, Edited by: Y. S. Touloukian. 

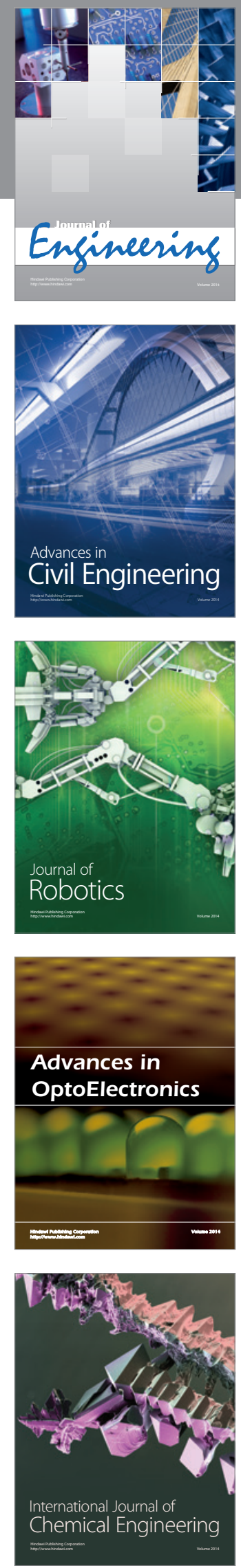

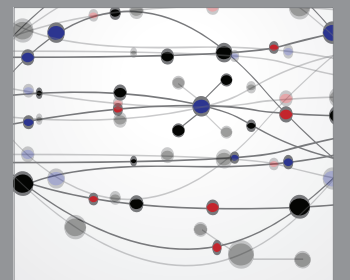

The Scientific World Journal
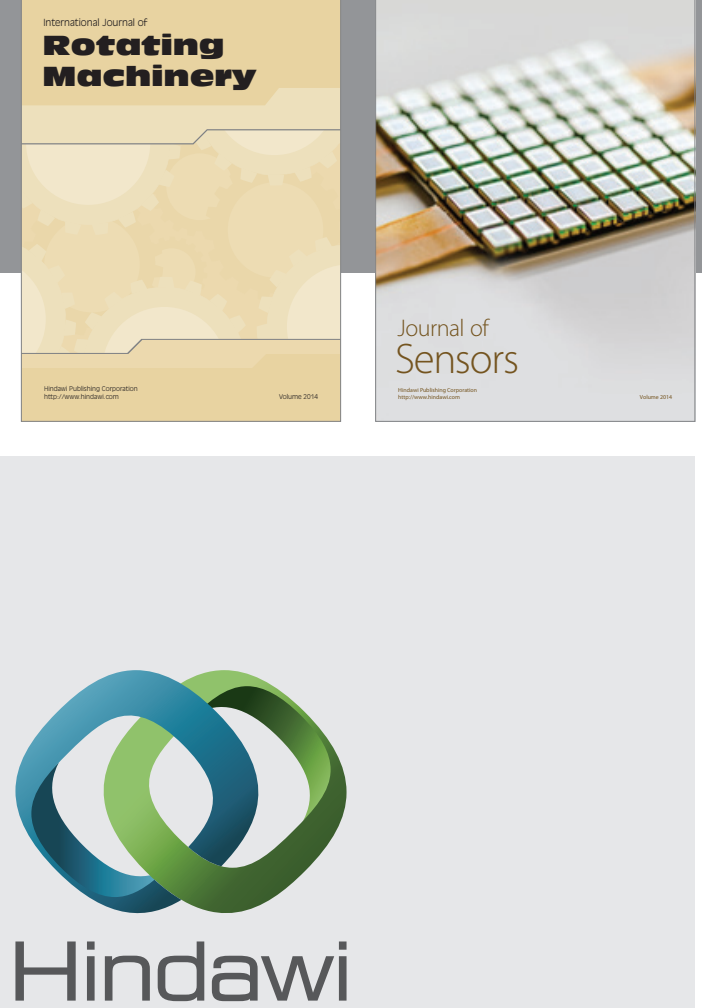

Submit your manuscripts at http://www.hindawi.com
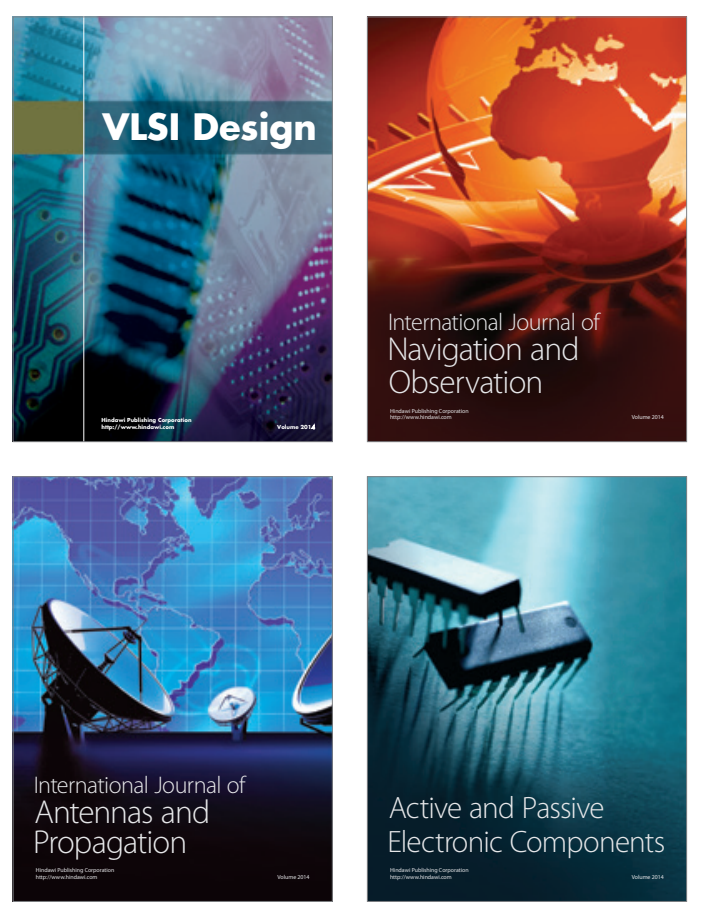
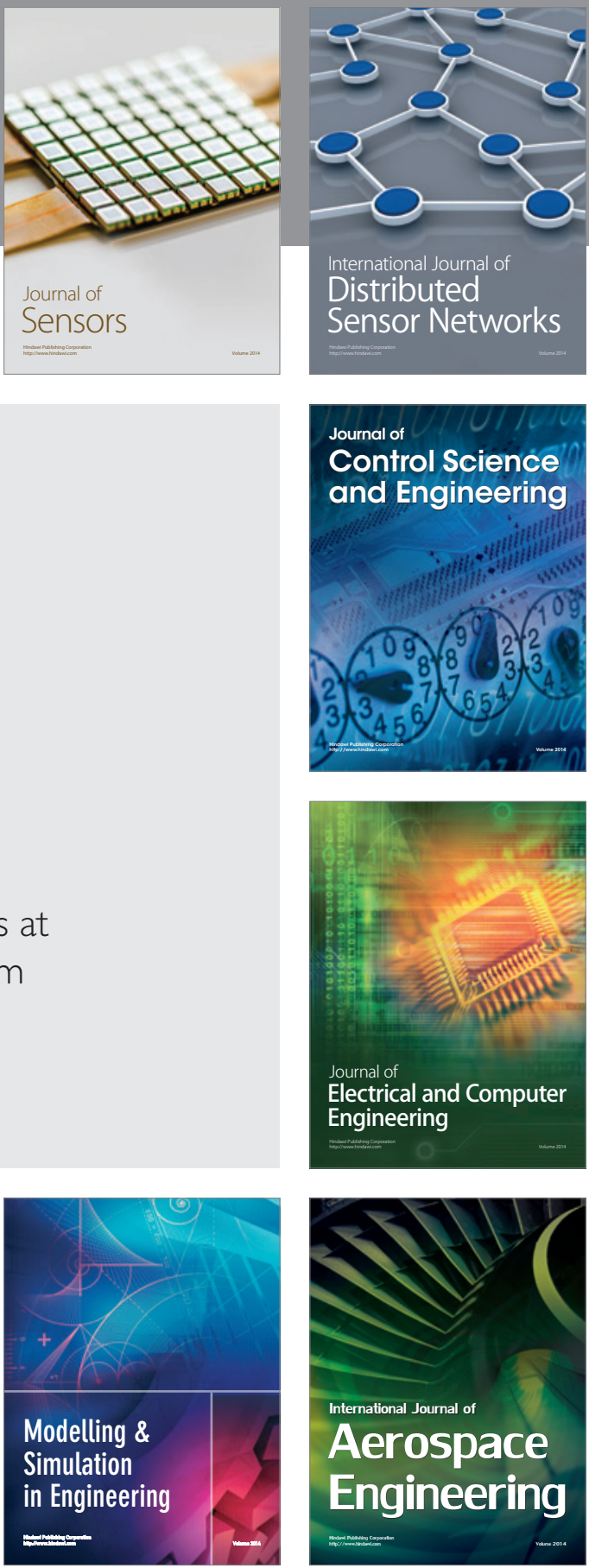

Journal of

Control Science

and Engineering
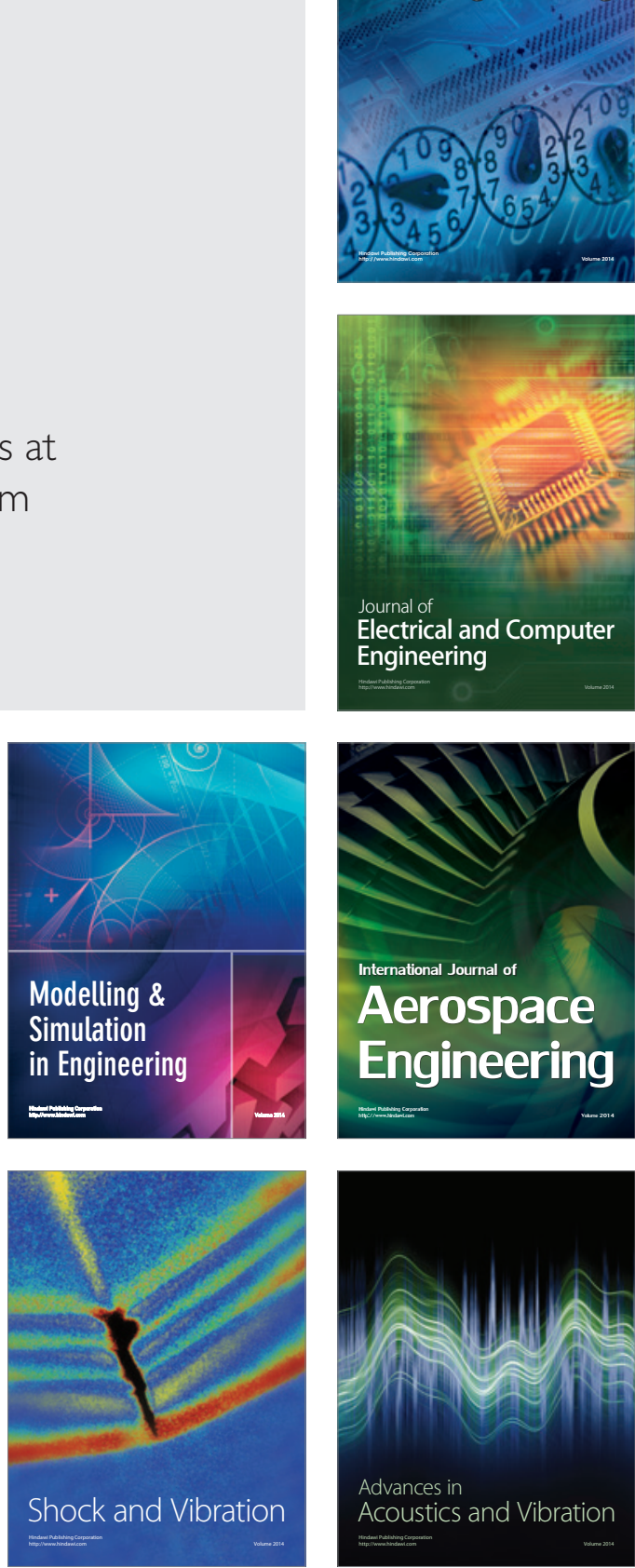